Check for updates

Cite this: RSC Adv., 2019, 9, 21063

Accepted 23rd May 2019

DOI: $10.1039 /$ c9ra03029h

rsc.li/rsc-advances

\section{Enantioselective Michael reaction of anthrone catalyzed by chiral tetraoxacalix[2]arene[2]triazine derivatives $\uparrow$}

\author{
Hayriye Nevin Genc (1D) * \\ A highly enantioselective Michael addition reaction of anthrone with nitroalkenes by chiral \\ tetraoxacalix[2]arene[2]triazine catalysts was investigated as a novel topic. The stereoselective \\ conversion progressed smoothly by employing $10 \mathrm{~mol} \%$ of the catalyst and afforded the \\ corresponding Michael adducts with acceptable to high enantioselectivities (up to 97\% ee) and very \\ high yields (up to $96 \%$ ).
}

\section{Introduction}

The chemical properties of anthrone and compounds containing the anthrone skeleton are significant in organic chemistry. Anthrones and their enol tautomers, i.e., 9anthrols form a vital part of anthracenes since the oxidation of the central rings yields 9,10-anthraquinones, while their reduction affords anthracenes, which are useful intermediates. ${ }^{1,2}$ However, naturally occurring compounds bearing the anthrone platform are isolated either as $\mathrm{O}-$ or Cglycosides or in a free form from a broad diversity of plants and shrubs such as rhubarb, cassia, and cascara sagrada. $^{3,4}$ Several such substances have noteworthy biological characteristics and are utilized as antimicrobial, emetic, antipsoriatic or androgen receptors and telomerase blockers. ${ }^{5,6}$ Recent studies have demonstrated that some anthrone- or anthraquinone-based naturally occurring compounds show strong and distinctive antitumor behaviours..$^{7-11}$

The Michael reaction of carbon-centered nucleophiles with different Michael acceptors provides a straightforward and robust technique for the formation of $\mathrm{C}-\mathrm{C}$ bonds and has received prevalent preference in the production of organic materials. As a result, substantial works have been carried out for the development of the enantioselective forms of this conversion. ${ }^{12-14}$ Although remarkable developments have emerged in the catalytic asymmetric Michael reaction, developing a new Michael reaction for the effective production of different novel materials remains a significant target for studies conducted in both academic and industrial contexts. In this

Department of Science Education, A. K. Education Faculty, Necmettin Erbakan University, Konya 42090, Turkey. E-mail: hngenc@erbakan.edu.tr; Fax: +90 332 3238225; Tel: +90 332 3238220/5534

$\dagger$ Electronic supplementary information (ESI) available. See DOI: 10.1039/c9ra03029h field of study, similar to the case of a Michael donor, various carbon-centered nucleophiles such as aldehydes and ketones, ${ }^{15-19}$ malonate esters, ${ }^{20-22}$ ketoesters, ${ }^{23}$ and 1,3-diketones ${ }^{24-26}$ have been comprehensively studied; in contrast, not much development has taken place in the improvement of the usage of anthrone as a nucleophile for the Michael addition reaction. ${ }^{27-33}$

Calixarenes and macromolecules bearing one or more calixarene platforms are known as efficient supramolecular materials. Heteroatom-bridged calixaromatics, also called heteroaromatic calixarenes, are a novel group of macrocyclic host compounds in supramolecular chemistry. ${ }^{34,35}$ Despite their exceptional physical and bonding characteristics that come from the electronic and steric influences of heteroatom bridges, heteroaromatic calixarenes are far rarer in usage..$^{36,37}$ The self-tuning and fine-tuning cavities of their electronic characteristics make heteroaromatic calixarenes strong macrocyclic hosts regarding their interactions with neutral organic guests $\mathrm{s}^{38,39}$ and with positively ${ }^{40}$ and negatively charged compounds. ${ }^{41-43}$ Oxygen- and nitrogen-bridged calix[2]arene[2] triazines are noteworthy heterocalixaromatics. ${ }^{44-47}$ Based on the characteristics of the heteroatoms in the bridging units, calix[2]arene[2]triazines use adaptable conformational constructs and may provide a diverse set of cavity sizes. Not limited to interactions of inclusion ${ }^{48}$ these materials may also show $\pi-\pi$ interactions of aromatic rings and hydrogen bonding interactions on the triazine nitrogen atoms as chiral host compounds.

In the past decade, we have reported the synthesis and applications of lower-rim-substituted calix[4]arene-based macromolecules with different functional groups as multiple $\mathrm{H}$-bond donor chiral catalysts for stereoselective conversions. ${ }^{49-51}$ Likewise, we have recently reported substituted tetraoxacalix[2]arene[2]triazine derivatives with different purposes as chiral catalysts in the stereoselective Michael additions of isobutyraldehyde using different substituted and 
unsubstituted aromatic trans- $\beta$-nitrostyrenes. ${ }^{52}$ Here, we described an efficient one-pot process to synthesize optically active tetraoxacalix[2]arene[2]triazine derivatives and their possible applications as organic catalysts in the Michael addition of anthrone to $\beta$-nitroolefins under mild conditions. As far as we know, this study is the first implementation of tetraoxacalix[2] arene[2]triazine catalysts in the enantioselective reactions of anthrone.

\section{Experimental}

\subsection{Synthesis}

2.1.1. Procedure for the synthesis of compounds $4 \mathrm{a}-4 \mathrm{~b}$. Tetraoxa-bridged calix[2] arene[2]triazine was prepared following a procedure described by Wang et al. ${ }^{53,54}$ A mixture of $(R)$-2-amino1-((S)-2-benzhydrylpyrrolidin-1-yl)-2-phenylethanone 1 or $(1 S, 2 S)$ $N, N$-bis(3-phenylpropyl)cyclohexane-1,2-diamine 2 (1.1 $\mathrm{mmol})$ and DIPEA $(2.2 \mathrm{mmol})$ in THF $(20 \mathrm{~mL})$ was added to a solution of tetraoxa-bridged calix[2] arene[2]triazine $3(0.5 \mathrm{mmol})$ in THF (20 $\mathrm{mL}$ ) at room temperature. The mixture was refluxed for $48-56 \mathrm{~h}$, after which the solvent was evaporated under vacuum, giving off a solid residue. The crude mixture was purified by column chromatography on silica gel using hexane/EtOAc $(10: 1, \mathrm{v} / \mathrm{v})$ to afford the desired products as crystalline solids. The products were characterized by a combination of ${ }^{1} \mathrm{H} \mathrm{NMR},{ }^{13} \mathrm{C} \mathrm{NMR}$, FTIR, ${ }^{55,56}$ and elemental analysis.

Compound 4a. Crystalline solid; 75\% yield; $\alpha_{\mathrm{D}}^{25}=-205.00$ (c 1, $\left.\mathrm{CHCl}_{3}\right)$; mp 220-222 ${ }^{\circ} \mathrm{C}$; IR $\left(\mathrm{cm}^{-1}\right): 1358,1479,1563$, 1708, 3261; ${ }^{1} \mathrm{H}$ NMR (400 $\left.\mathrm{MHz}, \mathrm{CDCl}_{3}\right): \delta=1.21-1.40(\mathrm{~m}$, $8 \mathrm{H}), 2.98-3.17(\mathrm{~m}, 4 \mathrm{H}), 4.72(\mathrm{~d}, 2 \mathrm{H}, J=6.7 \mathrm{~Hz}), 5.04(\mathrm{~d}, J=$ $6.8 \mathrm{~Hz}, 2 \mathrm{H}), 6.18(\mathrm{~s}, 2 \mathrm{H}), 7.16-7.38(\mathrm{~m}, 32 \mathrm{H}), 7.82-7.87(\mathrm{~m}$, $6 \mathrm{H})$, NH-signals not found; ${ }^{13} \mathrm{C}$ NMR $\left(100 \mathrm{MHz}, \mathrm{CDCl}_{3}\right): \delta=$ $23.34,29.47,46.29,51.17,58.64,65.80,124.74,127.33$, $127.63,128.00,128.23,128.43,128.60,128.73,129.05$, $129.68,130.00,138.20,140.53,140.68,158.20,162.20$, 166.00, 169.10; anal. calcd. for $\mathrm{C}_{68} \mathrm{H}_{58} \mathrm{~N}_{10} \mathrm{O}_{6}$ (1111.25): C, $73.49 ; \mathrm{H}, 5.26 ; \mathrm{N}, 12.60 \%$; found: C, 73.51; H, 5.31; N, $12.48 \%$.

Compound $4 \boldsymbol{b}$. Crystalline solid; $78 \%$ yield; $\alpha_{\mathrm{D}}^{25}=+218.00$ (c 1, $\left.\mathrm{CHCl}_{3}\right)$; $\mathrm{mp} 326-328^{\circ} \mathrm{C}$; IR $\left(\mathrm{cm}^{-1}\right): 1365,1483,1569,1705,3281$; ${ }^{1} \mathrm{H}$ NMR $\left(400 \mathrm{MHz}, \mathrm{CDCl}_{3}\right): \delta=1.19-1.35(\mathrm{~m}, 8 \mathrm{H}), 1.82-2.12(\mathrm{~m}$,
$16 \mathrm{H}), 2.60-2.75(\mathrm{~m}, 10 \mathrm{H}), 2.85-2.90(\mathrm{~m}, 8 \mathrm{H}), 4.13(\mathrm{q}, J=2.7 \mathrm{~Hz}$, $2 \mathrm{H}), 7.01-7.15(\mathrm{~m}, 20 \mathrm{H}), 7.20(\mathrm{t}, J=8.4,0.5 \mathrm{~Hz}, 2 \mathrm{H}), 7.25-7.30$ $(\mathrm{m}, 4 \mathrm{H}), 7.37-7.42(\mathrm{~m}, 2 \mathrm{H}), \mathrm{NH}$-signals not found; ${ }^{13} \mathrm{C} \mathrm{NMR}(100$ $\left.\mathrm{MHz}, \mathrm{CDCl}_{3}\right): \delta=14.16,21.03,27.51,32.04,32.50,34.14,57.00$, $60.47,62.33,102.86,116.90,128.41,128.90,128.96,130.28$, 140.40 , 157.07, 158.21, 166.00; anal. calcd. for $\mathrm{C}_{66} \mathrm{H}_{74} \mathrm{~N}_{10} \mathrm{O}_{4}$ (1071.35): C, 73.99; H, 6.96; N, 13.07\%; found: C, 74.15; H, 7.12; N, 12.98\%.

2.1.2. Procedure for Michael reaction. A solution of nitroalkenes $\mathbf{6 a}-\mathbf{k}(0.4 \mathrm{mmol})$ and the chiral catalyst $(0.04$ $\mathrm{mmol})$ in toluene $(4 \mathrm{~mL})$ was stirred and then, anthrone 5 $(0.48 \mathrm{mmol})$ was added. After being stirred for $36-72 \mathrm{~h}$ at room temperature, the reaction mixture was subjected directly to flash column chromatography on silica gel (hexane/EtOAc $=5: 1$ ) to furnish the corresponding products. The ee\% values of the Michael reaction products were determined by chiral HPLC analysis using Daicel Chiralpak OD-H or AS-H columns. The HPLC conditions for products 7a-7k are shown in Table 1 ; the ${ }^{1} \mathrm{H}$ NMR, ${ }^{13} \mathrm{C}$ NMR and FTIR spectroscopy values of products $7 \mathbf{a}-7 \mathbf{k}$ are shown in Table 2 .

\section{Results and discussion}

The chiral tetraoxacalix[2] arene[2]triazine derivatives $\mathbf{4 a}$ and $\mathbf{4 b}$, which were chosen as chiral catalysts in the model asymmetric Michael reaction, were synthesized in four steps starting from $(R)$-phenylglycine and $(1 S, 2 S)-(+)-1,2$-diaminocyclohexane, respectively. The chiral subunits $(R)$-2-amino-1- $((S)-2$ benzhydrylpyrrolidin-1-yl)-2-phenylethanone 1 and $(1 S, 2 S)-N, N$ bis(3-phenylpropyl) cyclohexane-1,2-diamine 2, which were synthesized in three steps, were prepared according to previously reported procedures, ${ }^{57,58}$ as illustrated in Scheme 1. Subsequently, the chiral tetraoxacalix[2] arene[2]triazines $\mathbf{4 a}$ and 4b were synthesized from 1 and 2, respectively, in overall good yields (up to $75-78 \%$ ), as illustrated in Scheme 2.

In the first review of the conditions, having selected the Michael addition of anthrone $\mathbf{5}$ and trans- $\beta$-nitrostyrene $\mathbf{6 a}$ as the enantioselective reaction, we found that the tetraoxacalix[2] arene[2]triazine catalysts catalyzed the process effectively, affording the expected adduct $7 \mathbf{a}$ in an optically active form. The reaction progressed successfully in nonpolar solvents including

Table 1 HPLC conditions of products $7 \mathrm{a}-7 \mathrm{k}$

\begin{tabular}{|c|c|c|c|c|c|}
\hline Product & Column & Hexane/2-propanol & Flow rate & $t_{\mathrm{R}}$ (minor) & $t_{\mathrm{R}}$ (major) \\
\hline $7 a$ & AS-H & $90: 10$ & $0.7 \mathrm{~mL} \mathrm{~min}^{-1}$ & $25.96 \mathrm{~min}$ & $23.25 \mathrm{~min}$ \\
\hline $7 \mathbf{b}$ & OD-H & $80: 20$ & $1.0 \mathrm{~mL} \mathrm{~min}^{-1}$ & $15.94 \mathrm{~min}$ & $12.85 \mathrm{~min}$ \\
\hline $7 \mathrm{c}$ & OD-H & $80: 20$ & $1.0 \mathrm{~mL} \min ^{-1}$ & $11.82 \mathrm{~min}$ & $10.06 \mathrm{~min}$ \\
\hline $7 d$ & AS-H & $80: 20$ & $1.0 \mathrm{~mL} \mathrm{~min}^{-1}$ & $12.85 \mathrm{~min}$ & $10.15 \mathrm{~min}$ \\
\hline $7 e$ & OD-H & $70: 30$ & $1.0 \mathrm{~mL} \mathrm{~min}{ }^{-1}$ & $18.14 \mathrm{~min}$ & $15.32 \mathrm{~min}$ \\
\hline $7 f$ & AS-H & $80: 20$ & $1.0 \mathrm{~mL} \mathrm{~min}^{-1}$ & $13.25 \mathrm{~min}$ & $10.42 \mathrm{~min}$ \\
\hline $7 g$ & AS-H & $80: 20$ & $1.0 \mathrm{~mL} \mathrm{~min}^{-1}$ & $17.43 \mathrm{~min}$ & $14.65 \mathrm{~min}$ \\
\hline $7 \mathbf{h}$ & AS-H & $90: 10$ & $1.0 \mathrm{~mL} \mathrm{~min}^{-1}$ & $18.19 \mathrm{~min}$ & $16.02 \mathrm{~min}$ \\
\hline $7 \mathbf{i}$ & OD-H & $80: 20$ & $1.0 \mathrm{~mL} \mathrm{~min}^{-1}$ & $19.62 \mathrm{~min}$ & $26.21 \mathrm{~min}$ \\
\hline $7 \mathbf{j}$ & AS-H & $90: 10$ & $1.0 \mathrm{~mL} \mathrm{~min}^{-1}$ & $26.11 \mathrm{~min}$ & $21.93 \mathrm{~min}$ \\
\hline $7 \mathbf{k}$ & AS-H & $70: 30$ & $1.0 \mathrm{~mL} \mathrm{~min} \min ^{-1}$ & $12.35 \mathrm{~min}$ & $10.19 \mathrm{~min}$ \\
\hline
\end{tabular}


Table $2{ }^{1} \mathrm{H}$ NMR, ${ }^{13} \mathrm{C}$ NMR, and FTIR spectroscopy values of products $7 \mathrm{a}-7 \mathrm{k}$

$\begin{array}{ll}7 a & \mathrm{Mp} \\ & \operatorname{IR}\left(\mathrm{cm}^{-1}\right) \\ & { }^{1} \mathrm{H} \text { NMR }\end{array}$

${ }^{1} \mathrm{H}$ NMR (400 MHz, $\left.\mathrm{CDCl}_{3}\right)$

${ }^{13} \mathrm{C}$ NMR $\left(100 \mathrm{MHz}, \mathrm{CDCl}_{3}\right)$

$7 \mathbf{b}$

Mp

$\operatorname{IR}\left(\mathrm{cm}^{-1}\right)$

${ }^{1} \mathrm{H}$ NMR $\left(400 \mathrm{MHz}, \mathrm{CDCl}_{3}\right)$

${ }^{13} \mathrm{C}$ NMR (100 MHz, $\left.\mathrm{CDCl}_{3}\right)$

$7 c$

Mp

IR $\left(\mathrm{cm}^{-1}\right)$

${ }^{1} \mathrm{H}$ NMR (400 MHz, $\mathrm{CDCl}_{3}$ )

${ }^{13} \mathrm{C}$ NMR (100 MHz, $\mathrm{CDCl}_{3}$ )

7d

Mp

IR $\left(\mathrm{cm}^{-1}\right)$

${ }^{1} \mathrm{H}$ NMR (400 MHz, $\mathrm{CDCl}_{3}$ )

${ }^{13} \mathrm{C}$ NMR (100 MHz, $\mathrm{CDCl}_{3}$ )

$7 e$

Mp

IR $\left(\mathrm{cm}^{-1}\right)$

${ }^{1} \mathrm{H}$ NMR (400 MHz, $\mathrm{CDCl}_{3}$ )

${ }^{13} \mathrm{C}$ NMR (100 MHz, $\mathrm{CDCl}_{3}$ )

$7 f$

Mp

IR $\left(\mathrm{cm}^{-1}\right)$

${ }^{1} \mathrm{H}$ NMR (400 MHz, $\mathrm{CDCl}_{3}$ )

${ }^{13} \mathrm{C}$ NMR (100 MHz, $\mathrm{CDCl}_{3}$ )

$7 \mathrm{~g}$

Mp

IR $\left(\mathrm{cm}^{-1}\right)$

${ }^{1} \mathrm{H}$ NMR (400 MHz, $\mathrm{CDCl}_{3}$ )

${ }^{13} \mathrm{C}$ NMR (100 MHz, $\mathrm{CDCl}_{3}$ )

$7 h$
Mp

IR $\left(\mathrm{cm}^{-1}\right)$

${ }^{1} \mathrm{H}$ NMR (400 MHz, $\mathrm{CDCl}_{3}$ )
$147-148{ }^{\circ} \mathrm{C}$

928, 1310, 1548, 1600, 1671

$4.03-4.06(\mathrm{~m}, 1 \mathrm{H}), 4.53(\mathrm{~d}, J=3.7 \mathrm{~Hz}, 1 \mathrm{H}), 4.60(\mathrm{dd}, J=13.3,7.0 \mathrm{~Hz}$, $1 \mathrm{H}), 4.88-4.91(\mathrm{dd}, J=13.3,9.1 \mathrm{~Hz}, 1 \mathrm{H}), 6.07(\mathrm{~d}, J=7.6 \mathrm{~Hz}, 2 \mathrm{H}), 6.91$ $(\mathrm{t}, J=7.8 \mathrm{~Hz}, 2 \mathrm{H}), 7.14-7.17(\mathrm{~m}, 1 \mathrm{H}), 7.39-7.43(\mathrm{~m}, 2 \mathrm{H}), 7.50(\mathrm{~d}, J=$ $8.5 \mathrm{~Hz}, 2 \mathrm{H}), 7.60-7.68(\mathrm{~m}, 2 \mathrm{H}), 7.94(\mathrm{~d}, J=7.8 \mathrm{~Hz}, 1 \mathrm{H}), 8.01(\mathrm{~d}, J=$ $8.0 \mathrm{~Hz}, 1 \mathrm{H})$

45.9, 52.8, 76.4, 126.1, 126.9, 127.8, 128.0, 128.1, 128.2, 128.3, 128.7, $131.9,132.2,132.9,133.4,134.6,139.8,142.3,183.1$ $65-67{ }^{\circ} \mathrm{C}$

$929,1317,1552,1598,1671$

4.31-4.39 (m, 2H), 4.59-4.69 (m, 2H), $6.18(\mathrm{~d}, J=8.5 \mathrm{~Hz}, 1 \mathrm{H}), 6.80(\mathrm{~d}$, $J=6.7 \mathrm{~Hz}, 1 \mathrm{H}), 7.00(\mathrm{~d}, J=7.1 \mathrm{~Hz}, 1 \mathrm{H}), 7.40-7.61(\mathrm{~m}, 4 \mathrm{H}), 7.63(\mathrm{~s}$, $2 \mathrm{H}), 8.13(\mathrm{t}, J=7.8 \mathrm{~Hz}, 2 \mathrm{H})$

45.0, 48.0, 74.0, 126.3, 127.1, 127.3, 127.9, 128.3, 128.5, 128.6, 129.8, 130.3, 131.7, 132.5, 133.0, 133.3, 133.6, 134.9, 136.0, 138.9, 140.2,

183.4

$141-143{ }^{\circ} \mathrm{C}$

$935,1322,1551,1600,1671$

$4.18-4.24(\mathrm{~m}, 1 \mathrm{H}), 4.37(\mathrm{dd}, J=13.3,8.4 \mathrm{~Hz}, 1 \mathrm{H}), 4.50(\mathrm{dd}, J=13.6$, $6.9 \mathrm{~Hz}, 1 \mathrm{H}), 4.68(\mathrm{~d}, J=3.6 \mathrm{~Hz}, 1 \mathrm{H}), 5.57(\mathrm{~d}, J=3.3 \mathrm{~Hz}, 1 \mathrm{H}), 6.20-6.22$ $(\mathrm{m}, 1 \mathrm{H}), 7.00(\mathrm{~d}, J=7.5 \mathrm{~Hz}, 1 \mathrm{H}), 7.19(\mathrm{~d}, J=1.1 \mathrm{~Hz}, 1 \mathrm{H}), 7.49-7.54(\mathrm{~m}$, $4 \mathrm{H}), 7.60-7.63(\mathrm{~m}, 1 \mathrm{H}), 8.20(\mathrm{t}, J=6.1 \mathrm{~Hz}, 2 \mathrm{H})$

44.8, 46.9, 73.9, 109.3, 110.1, 126.0, 127.3, 127.8, 128.2, 128.3, 128.5, 133.0, 133.2, 133.5, 133.7, 139.9, 140.5, 142.7, 148.4, 183.2

$63-65{ }^{\circ} \mathrm{C}$

$938,1320,1555,1601,1659$

4.00-4.03 (m, 1H), 4.81 (d, $J=5.4 \mathrm{~Hz}, 1 \mathrm{H}), 4.89-5.01(\mathrm{~m}, 1 \mathrm{H}), 5.12$ $(\mathrm{dd}, J=13.9,5.5 \mathrm{~Hz}, 1 \mathrm{H}), 6.60-6.62(\mathrm{~m}, 1 \mathrm{H}), 7.10-7.13(\mathrm{~m}, 2 \mathrm{H}), 7.29-$ $7.36(\mathrm{~m}, 2 \mathrm{H}), 7.51-7.65(\mathrm{~m}, 5 \mathrm{H}), 7.96(\mathrm{~d}, J=7.6 \mathrm{~Hz}, 1 \mathrm{H})$

45.0, 49.9, 77.1, 126.0, 126.1, 126.9, 127.8, 128.2, 129.0, 129.4, 130.0, $131.9,132.2,132.4,133.1,135.0,139.8,140.3,181.3$

$69-72{ }^{\circ} \mathrm{C}$

936, 1313, 1529, 1551, 1604, 1670

4.69-4.74 (m, 1H), $4.83(\mathrm{~d}, J=5.2 \mathrm{~Hz}, 1 \mathrm{H}), 5.09(\mathrm{dd}, J=13.6,10.3 \mathrm{~Hz}$, $1 \mathrm{H}), 5.30(\mathrm{dd}, J=13.9,5.5 \mathrm{~Hz}, 1 \mathrm{H}), 6.69-6.72(\mathrm{~m}, 1 \mathrm{H}), 7.29-7.38(\mathrm{~m}$, $3 \mathrm{H}), 7.48-7.62(\mathrm{~m}, 6 \mathrm{H}), 7.90(\mathrm{dd}, J=13.9,7.6 \mathrm{~Hz}, 2 \mathrm{H})$

44.9, 45.5, 76.8, 123.9, 125.8, 126.1, 127.6, 127.8, 128,2, 128.9, 129.1, 129.2, 129.5, 131.9, 132.0, 132.5, 132.9, 134.0, 140.0, 140.9, 150.0, 183.1

$129-131{ }^{\circ} \mathrm{C}$

929, 1320, 1509, 1555, 1600, 1661

3.19 (s, 1H), 4.39-4.43 (m, 1H), 4.69 (d, $J=3.9 \mathrm{~Hz}, 1 \mathrm{H}), 4.79$ (dd, $J=$ 13.1, $10.1 \mathrm{~Hz}, 1 \mathrm{H}), 5.09(\mathrm{dd}, J=13.4,5.9 \mathrm{~Hz}, 1 \mathrm{H}), 6.03(\mathrm{~d}, J=6.5 \mathrm{~Hz}$, $1 \mathrm{H}), 6.50(\mathrm{t}, J=7.4 \mathrm{~Hz}, 1 \mathrm{H}), 6.70(\mathrm{~d}, J=8.0 \mathrm{~Hz}, 1 \mathrm{H}), 7.10(\mathrm{t}, J=7.3 \mathrm{~Hz}$, $1 \mathrm{H}), 7.39-7.50(\mathrm{~m}, 4 \mathrm{H}), 7.59-7.62(\mathrm{t}, J=7.4 \mathrm{~Hz}, 2 \mathrm{H}), 7.82(\mathrm{~d}, J=$ $7.4 \mathrm{~Hz}, 1 \mathrm{H}), 7.89(\mathrm{~m}, J=7.9 \mathrm{~Hz}, 1 \mathrm{H})$

44.9, 45.0, 54.8, 76.7, 109.9, 120.4, 121.3, 124.6, 125.8, 126.2, 127.5, 128.0, 128.4, 129.0, 129.4, 131.9, 132.9, 141.0, 142.6, 156.9, 183.0 $115-117^{\circ} \mathrm{C}$

934, 1313, 1549, 1600, 1661

4.01-4.06 (m, 1H), 4.56-4.62 (m, 2H), $4.89(\mathrm{dd}, J=13.3,8.8 \mathrm{~Hz}, 1 \mathrm{H})$, $5.99(\mathrm{~d}, J=7.3 \mathrm{~Hz}, 1 \mathrm{H}), 6.17(\mathrm{~s}, 1 \mathrm{H}), 6.86(\mathrm{t}, J=7.9 \mathrm{~Hz}, 1 \mathrm{H}), 7.32(\mathrm{~d}, J$ $=9.1 \mathrm{~Hz}, 1 \mathrm{H}), 7.42-7.59(\mathrm{~m}, 4 \mathrm{H}), 7.65-7.71(\mathrm{~m}, 2 \mathrm{H}), 8.05(\mathrm{~d}, J=$ $7.6 \mathrm{~Hz}, 1 \mathrm{H}), 8.15(\mathrm{~d}, J=7.7 \mathrm{~Hz}, 1 \mathrm{H})$

45.9, 53.0, 76.1 121.9, 126.6, 127.2, 127.6, 128.3, 128.4, 128.5, 128.7, 129.6, 131.6, 131.9, 132.7, 133.0, 133.5, 134.6, 135.7, 139.0, 141.6, 183.0

$156-158{ }^{\circ} \mathrm{C}$

$929,1315,1548,1600,1659$

2.09 (s, 3H), 4.00-4.04 (m, 1H), 4.49 (d, $J=3.5 \mathrm{~Hz}, 1 \mathrm{H}), 4.54$ (dd, $J=$ 13.1, $7.1 \mathrm{~Hz}, 1 \mathrm{H}), 4.82$ (dd, $J=13.1,8.9 \mathrm{~Hz}, 1 \mathrm{H}), 5.99$ (d, $J=8.0 \mathrm{~Hz}$, $2 \mathrm{H}), 6.74(\mathrm{~d}, J=7.7 \mathrm{~Hz}, 2 \mathrm{H}), 7.35-7.45(\mathrm{~m}, 2 \mathrm{H}), 7.49(\mathrm{~d}, J=7.4 \mathrm{~Hz}$, $2 \mathrm{H}), 7.56-7.61(\mathrm{~m}, 2 \mathrm{H}), 8.03(\mathrm{~d}, J=7.7 \mathrm{~Hz}, 1 \mathrm{H}), 8.10(\mathrm{~d}, J=7.3 \mathrm{~Hz}$, $1 \mathrm{H})$ 
Table 2 (Contd.)

\begin{tabular}{|c|c|c|}
\hline & ${ }^{13} \mathrm{C}$ NMR $\left(100 \mathrm{MHz}, \mathrm{CDCl}_{3}\right)$ & $\begin{array}{l}20.9,45.9,53.1,75.8,126.4,126.9,127.4,127.9,128.3,128.5,128.6 \\
129.9,132.4,132.8,133.4,134.3,138.1,139.3,142.0,182.9\end{array}$ \\
\hline \multirow{4}{*}{$7 \mathbf{i}$} & Mp & $119-121{ }^{\circ} \mathrm{C}$ \\
\hline & $\operatorname{IR}\left(\mathrm{cm}^{-1}\right)$ & $929,1312,1511,1548,1599,1671$ \\
\hline & ${ }^{1} \mathrm{H}$ NMR $\left(400 \mathrm{MHz}, \mathrm{CDCl}_{3}\right)$ & $\begin{array}{l}3.71(\mathrm{~s}, 3 \mathrm{H}), 3.99-4.01(\mathrm{~m}, 1 \mathrm{H}), 4.49(\mathrm{~d}, J=3.8 \mathrm{~Hz}, 1 \mathrm{H}), 4.57(\mathrm{dd}, J= \\
13.3,7.4 \mathrm{~Hz}, 1 \mathrm{H}), 4.82(\mathrm{dd}, J=13.3,9.1 \mathrm{~Hz}, 1 \mathrm{H}), 6.00(\mathrm{~d}, J=8.8 \mathrm{~Hz}, \\
2 \mathrm{H}), 6.50(\mathrm{~d}, J=8.8 \mathrm{~Hz}, 2 \mathrm{H}), 7.40-7.54(\mathrm{~m}, 4 \mathrm{H}), 7.60-7.67(\mathrm{~m}, 2 \mathrm{H}), \\
8.02(\mathrm{~d}, J=7.9 \mathrm{~Hz}, 1 \mathrm{H}), 8.10(\mathrm{~d}, J=7.3 \mathrm{~Hz}, 1 \mathrm{H})\end{array}$ \\
\hline & ${ }^{13} \mathrm{C}$ NMR $\left(100 \mathrm{MHz}, \mathrm{CDCl}_{3}\right)$ & $\begin{array}{l}45.9,52.4,54.9,76.9,113.2,125.0,127.1,127.5,127.8,128.0,128.2 \\
128.3,129.4,132.6,132.8,133.9,134.8,139.0,141.9,160.0,182.9\end{array}$ \\
\hline \multirow[t]{4}{*}{$7 \mathbf{j}$} & Mp & $168-171^{\circ} \mathrm{C}$ \\
\hline & $\operatorname{IR}\left(\mathrm{cm}^{-1}\right)$ & $929,1321,1549,1600,1659$ \\
\hline & ${ }^{1} \mathrm{H}$ NMR $\left(400 \mathrm{MHz}, \mathrm{CDCl}_{3}\right)$ & $\begin{array}{l}4.01-4.04(\mathrm{~m}, 1 \mathrm{H}), 4.49(\mathrm{~d}, J=3.4 \mathrm{~Hz}, 1 \mathrm{H}), 4.60(\mathrm{dd}, J=13.3,7.6 \mathrm{~Hz} \text {, } \\
1 \mathrm{H}), 4.87(\mathrm{dd}, J=13.3,8.5 \mathrm{~Hz}, 1 \mathrm{H}), 5.99(\mathrm{~d}, J=8.5 \mathrm{~Hz}, 2 \mathrm{H}), 6.92(\mathrm{~d}, J= \\
8.5 \mathrm{~Hz}, 2 \mathrm{H}), 7.40-7.55(\mathrm{~m}, 4 \mathrm{H}), 7.61-7.67(\mathrm{~m}, 2 \mathrm{H}), 8.03(\mathrm{~d}, J=7.9 \mathrm{~Hz}, \\
1 \mathrm{H}), 8.09(\mathrm{~d}, J=7.6 \mathrm{~Hz}, 1 \mathrm{H})\end{array}$ \\
\hline & ${ }^{13} \mathrm{C}$ NMR $\left(100 \mathrm{MHz}, \mathrm{CDCl}_{3}\right)$ & $\begin{array}{l}45.8,52.5,76.4,126.8,127.0,127.2,127.6,127.9,128.1,128.2,128.4 \\
130.0,131.9,132.2,132.9,133.1,134.0,134.5,138.9,141.7,182.9\end{array}$ \\
\hline \multirow[t]{4}{*}{$7 \mathbf{k}$} & Mp & $171-172{ }^{\circ} \mathrm{C}$ \\
\hline & $\operatorname{IR}\left(\mathrm{cm}^{-1}\right)$ & $929,1323,1511,1548,1605,1658$ \\
\hline & ${ }^{1} \mathrm{H}$ NMR $\left(400 \mathrm{MHz}, \mathrm{CDCl}_{3}\right)$ & $\begin{array}{l}4.01-4.06(\mathrm{~m}, 1 \mathrm{H}), 4.55(\mathrm{~d}, J=3.7 \mathrm{~Hz}, 1 \mathrm{H}), 4.60(\mathrm{dd}, J=13.3,7.3 \mathrm{~Hz}, \\
1 \mathrm{H}), 4.90(\mathrm{dd}, J=13.3,9.1 \mathrm{~Hz}, 1 \mathrm{H}), 5.99(\mathrm{dd}, J=8.8,5.2 \mathrm{~Hz}, 2 \mathrm{H}), 6.67 \\
(\mathrm{t}, J=8.8 \mathrm{~Hz}, 2 \mathrm{H}), 7.45-7.58(\mathrm{~m}, 4 \mathrm{H}), 7.64-7.68(\mathrm{~m}, 2 \mathrm{H}), 7.98(\mathrm{~d}, J= \\
7.3 \mathrm{~Hz}, 1 \mathrm{H}), 8.07(\mathrm{~d}, J=7.9 \mathrm{~Hz}, 1 \mathrm{H})\end{array}$ \\
\hline & ${ }^{13} \mathrm{C}$ NMR $\left(100 \mathrm{MHz}, \mathrm{CDCl}_{3}\right)$ & $\begin{array}{l}46.3,52.8,76.6,114.8,115.2,127.1,127.6,127.9,128.0,128.2,128.5 \text {, } \\
128.9,129.5,130.8,131.9,132.8,133.6,134.5,138.9,142.1,161.9, \\
182.9\end{array}$ \\
\hline
\end{tabular}

hexane, $\mathrm{CHCl}_{3}, \mathrm{CH}_{2} \mathrm{Cl}_{2}$ or toluene (Table 3, entries 1-8); however, when we used a polar solvent, such as ethyl acetate, diethyl ether or acetone, significant decrease was observed in chemical yield and enantioselectivity (Table 3, entries 15-20). This may be due to the fact that the polar solvents interacted with the organocatalysts through hydrogen bonding to weaken the activation ability of $\mathbf{4 a}$ and $\mathbf{4 b}$ towards the reaction. As projected by our tentative hypothesis, the best yield (96\%) and enantioselectivity ( $97 \%$ ee) were obtained using the less polar solvent, i.e., toluene.
Other aspects of this reaction such as temperature, use of the recycled catalyst, and catalyst loading were investigated (Table 4). When the same reaction was performed at $0{ }^{\circ} \mathrm{C}$ with $10 \mathrm{~mol} \%$ of $\mathbf{4 a}$ and $\mathbf{4 b}$ as catalysts, the desired adduct 7a was obtained with low to $88-89 \%$ ee in $83-85 \%$ yield, with further extension of the reaction time ( $72 \mathrm{~h}$, Table 4 , entries 3 and 4 ). In addition, similar to the reaction conducted at $0{ }^{\circ} \mathrm{C}$, the reaction carried out at $-20{ }^{\circ} \mathrm{C}$ showed less desired yields and enantioselectivities than the reaction at room temperature (Table 4, entries 1 and 2). Additionally, we conducted recycling analysis of the chiral catalysts $\mathbf{4 a}$ and $\mathbf{4 b}$ in the Michael

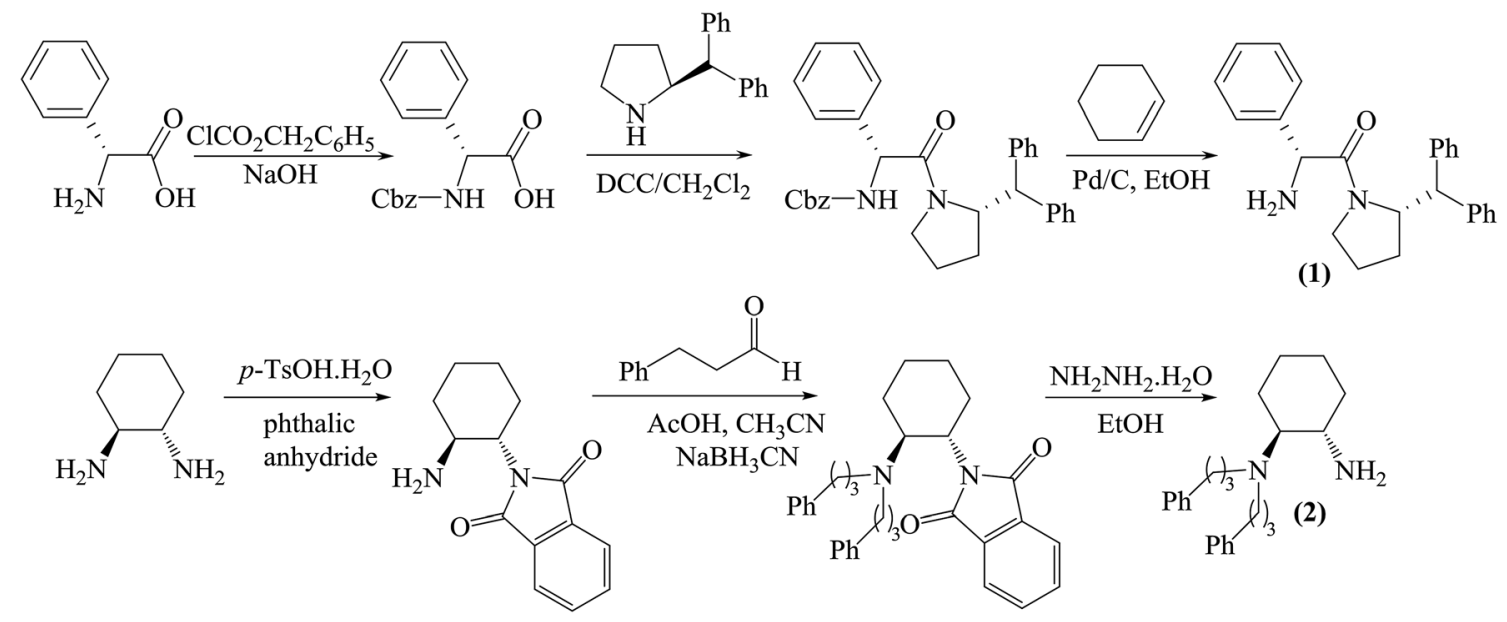

Scheme 1 The synthetic routes for starting materials 1 and 2 . 


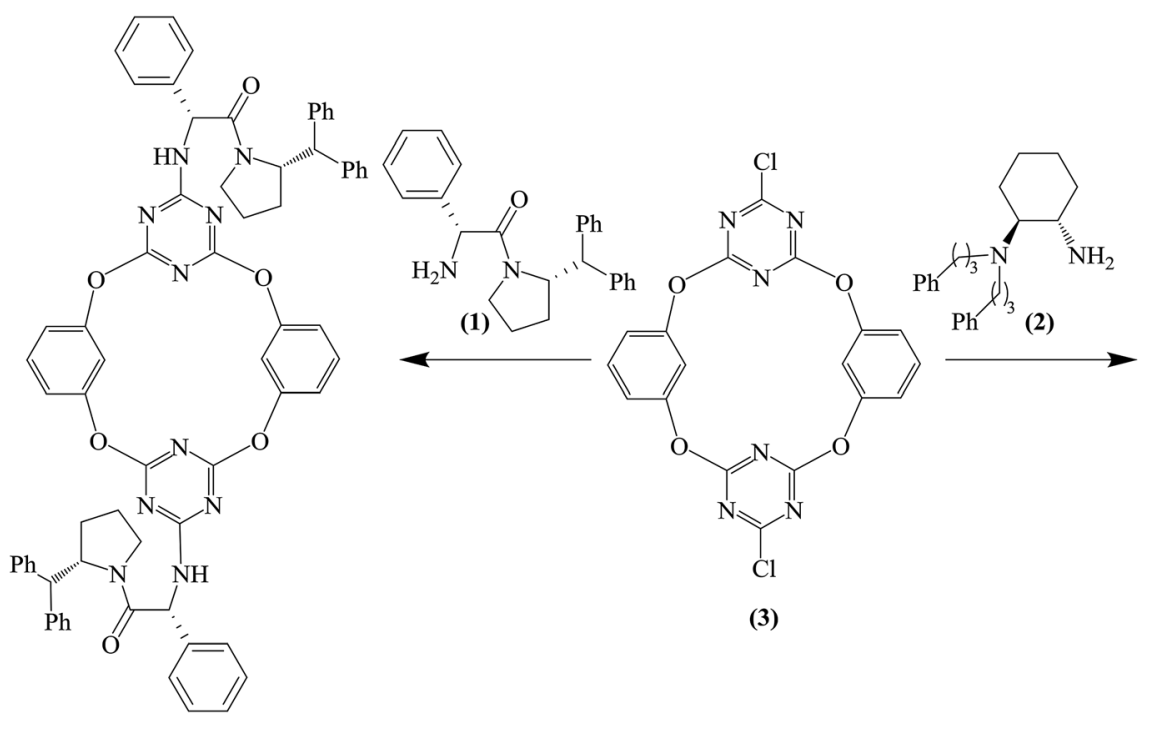

(4a)

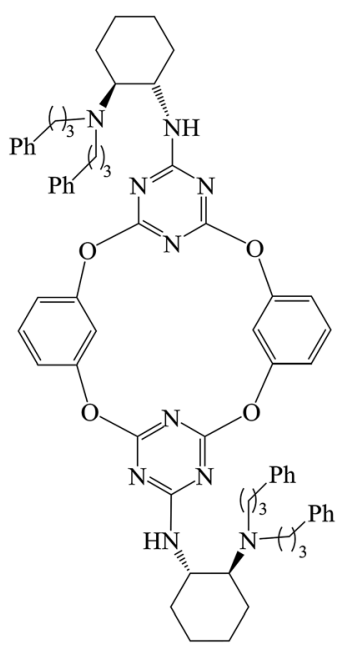

(4b)

Scheme 2 The synthetic route for tetraoxa-bridged calix[2]arene[2]triazine derivatives 4a/4b.

reaction of $\mathbf{5}$ and $6 \mathbf{a}$. Thus, the compounds $4 \mathbf{a}$ and $4 \mathbf{b}$ could be easily recycled by flash chromatography alongside Michael

Table 3 Michael addition between anthrone 5 and trans- $\beta$-nitrostyrene $6 a$ catalysed by $4 a-4 b$ in various solvents<smiles>O=C1c2ccccc2Cc2ccccc21</smiles>

(5)

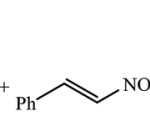

(6a)

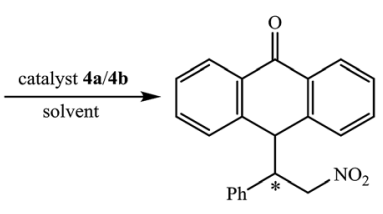

(7a)

\begin{tabular}{|c|c|c|c|c|c|}
\hline Entry $^{a}$ & Catalyst & Solvent & Time (h) & Yield $^{b}(\%)$ & $\mathrm{ee}^{c, d}(\%)$ \\
\hline 1 & $4 a$ & Hexane & 72 & 88 & 93 \\
\hline 2 & $4 b$ & Hexane & 72 & 90 & 95 \\
\hline 3 & $4 a$ & $\mathrm{CHCl}_{3}$ & 48 & 85 & 90 \\
\hline 4 & $4 b$ & $\mathrm{CHCl}_{3}$ & 48 & 89 & 91 \\
\hline 5 & $4 a$ & $\mathrm{CH}_{2} \mathrm{Cl}_{2}$ & 48 & 81 & 92 \\
\hline 6 & $4 b$ & $\mathrm{CH}_{2} \mathrm{Cl}_{2}$ & 48 & 83 & 93 \\
\hline 7 & $4 a$ & Toluene & 48 & 95 & 96 \\
\hline 8 & $4 b$ & Toluene & 48 & 96 & 97 \\
\hline 9 & $4 a$ & $\mathrm{CH}_{3} \mathrm{CN}$ & 72 & 92 & 80 \\
\hline 10 & $4 b$ & $\mathrm{CH}_{3} \mathrm{CN}$ & 72 & 95 & 81 \\
\hline 11 & $4 a$ & Xylene & 72 & 87 & 91 \\
\hline 12 & $4 b$ & Xylene & 72 & 90 & 95 \\
\hline 13 & $4 a$ & THF & 72 & 82 & 84 \\
\hline 14 & $4 b$ & $\mathrm{THF}$ & 72 & 85 & 87 \\
\hline 15 & $4 a$ & EtOAc & 48 & 87 & 80 \\
\hline 16 & $4 b$ & EtOAc & 48 & 91 & 85 \\
\hline 17 & $4 a$ & $\mathrm{Et}_{2} \mathrm{O}$ & 72 & 83 & 88 \\
\hline 18 & $4 b$ & $\mathrm{Et}_{2} \mathrm{O}$ & 72 & 86 & 89 \\
\hline 19 & $4 a$ & Acetone & 48 & 85 & 82 \\
\hline 20 & $4 b$ & Acetone & 48 & 88 & 85 \\
\hline
\end{tabular}

${ }^{a}$ Conditions: anthrone $(0.48 \mathrm{mmol})$, trans- $\beta$-nitrostyrene $(0.40 \mathrm{mmol})$ and $4 \mathbf{4 a} / \mathbf{4 b}(10 \mathrm{~mol} \%)$ in solvents $(4.0 \mathrm{~mL}) \cdot{ }^{b}$ Isolated yield after flash chromatograpy. ${ }^{c}$ Determined by HPLC. ${ }^{d}$ Determined by comparing reported data. adducts. However, by prolonging the reaction time, low enantioselectivity and yield values were found at room temperature, as seen in Table 4 (entries 7 and 8). In the presence of $10 \mathrm{~mol} \%$ catalysts, $\mathbf{4 a}$ and $\mathbf{4 b}$ showed similar enantioselectivities, but $\mathbf{4 b}$ showed slightly higher catalytic activity than $4 \mathbf{a}$ (Table 4 , entries 5 and 6 ). When the loading of the catalysts $\mathbf{4 a}$ and $\mathbf{4 b}$ went up to $15 \mathrm{~mol} \%$, trans- $\beta$ nitrostyrene gave Michael products in low yields with 93-95\% and $93-95 \%$ enantiomeric excess, respectively (Table 4, entries 9 and 10). These results were better than that for the use of $5 \mathrm{~mol} \%$ of catalyst, in which case the yields of the Michael products were $88 \%$ for catalyst $4 \mathrm{a}$ and $90 \%$ for catalyst $\mathbf{4} \mathbf{b}$ and the enantiomeric excesses of the Michael products were $89 \%$ for catalyst $\mathbf{4 a}$ and $90 \%$ for catalyst $\mathbf{4 b}$ (Table 4, entries 11 and 12). These findings led us to choose the reaction conditions using toluene as a solvent at room temperature in the presence of $10 \mathrm{~mol} \%$ of $\mathbf{4 b}$ to probe the scope of nitroolefins.

As we enhanced the reaction details for the Michael addition of anthrone 5 to trans- $\beta$-nitrostyrene $\mathbf{6 a}$ (catalyst $\mathbf{4 b}$ $10 \mathrm{~mol} \%$ in toluene at room temperature), a set of different nitrostyrenes with various substituent groups were analyzed, and the results are summarized in Table 5. These nitrostyrenes reacted with anthrone to afford the corresponding adducts $\mathbf{7 a - 7 k}$ in moderate to excellent yields with excellent enantioselectivities (Table 5, entries 1-11). As demonstrated in Table 5, anthrone reacts smoothly with a wide range of ortho-, meta- or para-substituted nitrostyrenes with electronreleasing or electron-withdrawing groups and the corresponding Michael adducts in good to excellent yields (86$96 \%$ ) and enantioselectivities (81-97\%) are obtained. To our satisfaction, trans- $\beta$-nitrostyrene and 4-Me-nitrostyrene as Michael acceptors gave good yields and enantioselectivities (Table 5, entries 1 and 8).

In conclusion, we developed highly efficient asymmetric Michael addition of anthrone to nitroalkenes catalyzed by 
Table 4 Screening of various reaction conditions

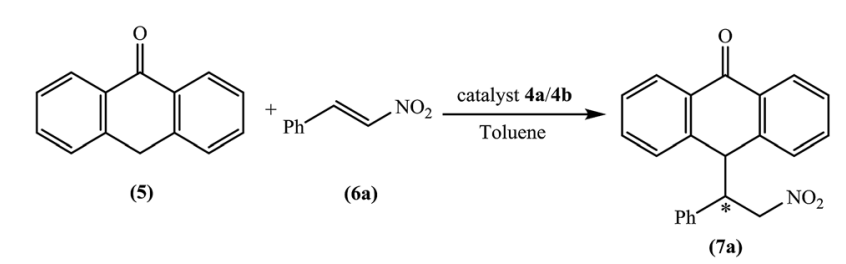

\begin{tabular}{|c|c|c|c|c|c|}
\hline Entry $^{a}$ & Catalyst & $\begin{array}{l}\text { Temp. } \\
\left({ }^{\circ} \mathrm{C}\right)\end{array}$ & Time (h) & Yield $^{b}(\%)$ & $\mathrm{ee}^{c, d}(\%)$ \\
\hline 1 & $4 a$ & -20 & 72 & 80 & 85 \\
\hline 2 & $4 b$ & -20 & 72 & 81 & 86 \\
\hline 3 & $4 a$ & 0 & 72 & 83 & 88 \\
\hline 4 & $4 \mathrm{~b}$ & 0 & 72 & 85 & 89 \\
\hline 5 & $4 a$ & r.t. & 48 & 95 & 96 \\
\hline 6 & $4 b$ & r.t. & 48 & 96 & 97 \\
\hline $7^{e}$ & $4 a$ & r.t. & 72 & 75 & 86 \\
\hline $8^{e}$ & $4 b$ & r.t. & 72 & 78 & 90 \\
\hline $9^{f}$ & $4 a$ & r.t. & 48 & 93 & 93 \\
\hline $10^{f}$ & $4 b$ & r.t. & 48 & 95 & 95 \\
\hline $11^{g}$ & $4 a$ & r.t. & 48 & 88 & 89 \\
\hline $12^{g}$ & $4 b$ & r.t. & 48 & 90 & 90 \\
\hline
\end{tabular}

${ }^{a}$ Conditions: anthrone $(0.48 \mathrm{mmol})$, trans- $\beta$-nitrostyrene $(0.40 \mathrm{mmol})$, and $4 \mathbf{a} / \mathbf{4 b}(10 \mathrm{~mol} \%)$ in toluene $(4.0 \mathrm{~mL}) .{ }^{b}$ Isolated yield. ${ }^{c}$ Determined by HPLC. ${ }^{d}$ Determined by comparing reported data. ${ }^{e}$ Reaction was performed with recycled catalyst. ${ }^{f} 15 \mathrm{~mol} \%$ of catalyst was used. ${ }^{g} 5 \mathrm{~mol} \%$ of catalyst was used.

tetraoxa-bridged calix[2] arene[2]triazine organocatalysts. The results of our study were similar to the literatures which were used thiourea-tertiary amine, ${ }^{32}$ cinchona alkaloids, ${ }^{33}$ cinchona-

Table 5 Scope of the Michael addition with anthrone 5 and nitroalkenes $6 \mathrm{a}-\mathrm{k}$

$$
\text { (5) }
$$

\begin{tabular}{llllll} 
Entry $^{a}$ & Ar & Time (h) & Product & Yield $^{b}(\%)$ & ee $^{c, d}(\%)$ \\
\hline 1 & $\mathrm{C}_{6} \mathrm{H}_{5}$ & 48 & $\mathbf{7 a}$ & 96 & 97 \\
2 & $2,4-\mathrm{Cl}_{2}-\mathrm{C}_{6} \mathrm{H}_{4}$ & 36 & $7 \mathbf{b}$ & 88 & 82 \\
3 & $2-\mathrm{Furyl}^{-} \mathrm{C}_{6} \mathrm{H}_{4}$ & 48 & $7 \mathbf{c}$ & 94 & 88 \\
4 & $2-\mathrm{Br}_{-} \mathrm{C}_{6} \mathrm{H}_{4}$ & 36 & $7 \mathbf{d}$ & 94 & 81 \\
5 & $2-\mathrm{NO}_{2}-\mathrm{C}_{6} \mathrm{H}_{4}$ & 48 & $7 \mathbf{e}$ & 91 & 89 \\
6 & $2-\mathrm{OMe}-\mathrm{C}_{6} \mathrm{H}_{4}$ & 48 & $7 \mathbf{f}$ & 86 & 95 \\
7 & $3-\mathrm{Br}_{-} \mathrm{C}_{6} \mathrm{H}_{4}$ & 36 & $7 \mathbf{g}$ & 93 & 96 \\
8 & $4-\mathrm{Me}-\mathrm{C}_{6} \mathrm{H}_{4}$ & 48 & $7 \mathbf{h}$ & 95 & 97 \\
9 & $4-\mathrm{OMe}-\mathrm{C}_{6} \mathrm{H}_{4}$ & 48 & $7 \mathbf{i}$ & 95 & 91 \\
10 & $4-\mathrm{Cl}-\mathrm{C}_{6} \mathrm{H}_{4}$ & 36 & $7 \mathbf{j}$ & 91 & 82 \\
11 & $4-\mathrm{F}-\mathrm{C}_{6} \mathrm{H}_{4}$ & 36 & $7 \mathbf{k}$ & 91 & 92
\end{tabular}

${ }^{a}$ Conditions: anthrone $(0.48 \mathrm{mmol})$, trans- $\beta$-nitrostyrene $(0.40 \mathrm{mmol})$, and $4 \mathbf{b}(10 \mathrm{~mol} \%)$ in toluene $(4.0 \mathrm{~mL}) .{ }^{b}$ Isolated yield after flash chromatograpy. ${ }^{c}$ Determined by HPLC. ${ }^{d}$ The configurations were determined by comparing reported data. based chiral polyesters, ${ }^{59}$ and Ar-BINMOLs ${ }^{60}$ catalysts for the reaction of anthrone to a series of nitroalkenes. The steric bulkiness and carbonyl groups were crucial in this reaction to give the corresponding adducts in lower ee than that for the catalyst without carbonyl groups. Efforts to elucidate the mechanistic details of this catalytic system and to further extend the scope and limitations of these kinds of organocatalysts are currently in progress.

\section{Conclusions}

In conclusion, in this work, a new class of chiral tetraoxabridged calix[2]arene[2]triazine derivatives described as effective organocatalysts for the Michael reaction of anthrone to various nitrostyrenes was reported for the first time. The addition reactions were carried out smoothly in toluene at room temperature by utilizing $10 \mathrm{~mol} \%$ of $\mathbf{4 a}$ and $\mathbf{4 b}$ to give Michael products with high yields (up to $96 \%$ ) and ee values (up to 97\%).

\section{Conflicts of interest}

There are no conflicts to declare.

\section{Notes and references}

1 K. Srinivas, K. Yesudas, K. Bhanuprakash, J. V. Rao and L. A. Giribabu, J. Phys. Chem. C, 2009, 113, 20117-20126.

2 R. Iwaura, M. Ohnishi-Kameyama and T. Lizawa, Chem.-Eur. J., 2009, 15, 3729-3735.

3 L. Krenn, R. Pradhan, A. Presser, G. Reznicek and B. Kopp, Chem. Pharm. Bull., 2004, 52, 391-393.

4 F. Diaz, H.-B. Chai, Q. Mi, B.-N. Su, J. S. Vigo, J. G. Graham, F. Cabieses, N. R. Farnsworth, G. A. Cordell, J. M. Pezzuto, S. M. Swanson and A. D. Kinghorn, J. Nat. Prod., 2004, 67, 352-356.

5 D. W. Cameron and C. E. Skene, Aust. J. Chem., 1996, 49, 617624.

6 H.-S. Huang, J.-M. Hwang, Y.-M. Jen, J.-J. Lin, K.-Y. Lee, C.-H. Shi and H.-C. Hsu, Chem. Pharm. Bull., 2001, 49, 969973.

7 V. Kren and T. Rezanka, FEMS Microbiol. Rev., 2008, 32, 858889.

8 K. Müuller, Appl. Microbiol. Biotechnol., 2001, 56, 9-16.

9 T. Pecere, M. V. Gazzola, C. Mucignat, C. Parolin, F. D. Vecchia, A. Cavaggini, G. Basso, A. Diaspro, B. Salvato, M. Carli and G. Pulci, Cancer Res., 2000, 60, 2800-2804.

10 Y. Shiono, N. Shino, S. Seo, S. Oka and Y. Z. Yamazaki, Naturforscher, 2002, 57c, 923-929.

11 A. Zuse, D. Schmidt, S. Baasner, K. J. Büohm, K. Müuller, M. Gerlach, E. G. Güunther, E. Unger and H. Prinz, J. Med. Chem., 2007, 50, 6059-6066.

12 B. M. Trost, Acc. Chem. Res., 2002, 35, 695-705.

13 O. M. Berner, L. Tedeschi and D. Enders, Eur. J. Org. Chem., 2002, 1877-1894.

14 D. Almasi, D. A. Alonso and C. Najera, Tetrahedron: Asymmetry, 2007, 18, 299-365. 
15 H. Huang and E. N. Jacobsen, J. Am. Chem. Soc., 2006, 128, 7170-7171.

16 M. P. Lalonde, Y. Chen and E. N. Jacobsen, Angew. Chem., Int. Ed., 2006, 45, 6366-6370.

17 W. Wang, J. Wang and H. Li, Angew. Chem., Int. Ed., 2005, 43, 1369-1371.

18 Y. Hayashi, T. Gotoh, T. Hayasji and M. Shoji, Angew. Chem., Int. Ed., 2005, 44, 4212-4215.

19 T. Ishii, S. Fiujioka, Y. Sekiguchi and H. Kotsuki, J. Am. Chem. Soc., 2004, 126, 9558-9559.

20 Y. Li, H. Wang, L. Tang and L. Deng, J. Am. Chem. Soc., 2004, 126, 9906-9907.

21 T. Okino, Y. Hoashi, T. Furukawa, X. Xu and Y. Takemoto, J. Am. Chem. Soc., 2005, 127, 119-125.

22 M. Terada, H. Ube and Y. Yaguchi, J. Am. Chem. Soc., 2006, 128, 1454-1455.

23 H. Li, Y. Wang, L. Tang, F. Wu, X. Liu, C. Guo, B. M. Foxman and L. Deng, Angew. Chem., Int. Ed., 2005, 44, 105-108.

24 J. Wang, H. Li, W. Duan, L. Zu and W. Wang, Org. Lett., 2005, 7, 4713-4716.

25 F.-Z. Peng, Z.-H. Shao, B.-M. Fan, H. Song, G.-P. Li and H.-B. Zhang, J. Org. Chem., 2008, 73, 5202-5205.

26 P. Gao, C. Wang, Y. Wu, Z. Zhou and C. Tang, Eur. J. Org. Chem., 2008, 4563-4566.

27 J. Shen, T. T. Nguyen, Y.-P. Goh, W. Ye, X. Fu, J. Xu and C.-H. Tan, J. Am. Chem. Soc., 2006, 128, 13692-13693.

28 A.-N. Alba, N. Bravo, A. Moyano and R. Rios, Tetrahedron Lett., 2009, 50, 3067-3069.

29 D. Akalay, G. Durner and M. W. Gobel, Eur. J. Org. Chem., 2008, 2365-2368.

30 C. Wu, W. Li, J. Yang, X. Liang and J. Ye, Org. Biomol. Chem., 2010, 8, 3244-3250.

31 A. Zea, G. Valero, A. N. R. Alba, A. Moyano and R. Rios, Adv. Synth. Catal., 2010, 352, 1102-1106.

32 Y.-H. Liao, H. Zhang, Z.-J. Wu, L.-F. Cun, X.-M. Zhang and W.-C. Yuan, Tetrahedron: Asymmetry, 2009, 20, 2397-2402.

33 M. Shi, Z.-Y. Lei, M.-X. Zhao and J.-W. Shi, Tetrahedron Lett., 2007, 48, 5743-5746.

34 M. M. Naseer, D. X. Wang, L. Zhao, Z. T. Huang and M. X. Wang, J. Org. Chem., 2011, 76, 1804-1813.

35 S. Li, D. X. Wang and M. X. Wang, Tetrahedron Lett., 2012, 53, 6426-6429.

36 A. I. Vicente, J. M. Caio, J. Sardinha, C. Oiteiro, R. Delgado and V. Felix, Tetrahedron, 2012, 68, 670-680.

37 L. X. Wang, L. Zhao and D. X. Wang, Chem. Commun., 2011, 9690-9692.
38 M. H. Duker, H. Schafer, M. Zeller and V. A. Azov, J. Org. Chem., 2013, 78, 4905-4912.

39 W. Zhao, K. Hu, C. Wang, S. Liang, B. Niu, L. He, K. Lu, B. Ye and S. Zhang, J. Chromatogr. A, 2012, 1223, 72-78.

40 H. B. Yang, D. X. Wang, Q. Q. Wang and M. X. Wang, J. Org. Chem., 2007, 72, 3757-3763.

41 D. X. Wang, Q. Y. Zheng, Q. Q. Wang and M. X. Wang, Angew. Chem., Int. Ed., 2008, 47, 7485-7488.

42 D. X. Wang, S. X. Fa, Y. Liu, B. Y. Hou and M. X. Wang, Chem. Commun., 2012, 11458-11460.

43 S. Pan, D. X. Wang, L. Zhao and M. X. Wang, Tetrahedron, 2012, 68, 9464-9477.

44 M.-X. Wang, Acc. Chem. Res., 2012, 45, 182-195.

45 W. Maes and W. Dehaen, Chem. Soc. Rev., 2008, 37, 23932402.

46 H. Tsue, K. Ishibashi and R. Tamura, Top. Heterocycl. Chem., 2008, 17, 73-96.

47 X. D. Wang, D. X. Wang, Z. T. Huang and M. X. Wang, Supramol. Chem., 2014, 26, 601-606.

48 W. Zhao, W. Wang, H. Chang, S. Cui, K. Hu, L. He, K. Lu, J. Liu, W. Wu, J. Qian and S. Zhang, J. Chromatogr. A, 2012, 1251, 74-81.

49 H. N. Naziroglu and A. Sirit, Tetrahedron: Asymmetry, 2016, 27, 201-207.

50 H. N. Genc and A. Sirit, J. Inclusion Phenom. Macrocyclic Chem., 2018, 90, 39-49.

51 H. N. Naziroglu, M. Durmaz, S. Bozkurt, A. S. Demir and A. Sirit, Tetrahedron: Asymmetry, 2012, 23, 164-169.

52 H. N. Genc, U. Ozgun and A. Sirit, Chirality, 2019, 31, 293300.

53 M.-X. Wang and H.-B. Yang, J. Am. Chem. Soc., 2004, 126, 15412-15422.

54 S. Bozkurt and M. B. Türkmen, Tetrahedron: Asymmetry, 2016, 27, 443-447.

55 Y. Huangfu, K. Ruan, H. Qiu, Y. Lu, C. Liang, J. Kong and J. Gu, Composites, Part A, 2019, 121, 265-272.

56 C. Liang, H. Qiu, Y. Han, H. Gu, P. Song, L. Wang, J. Kong, D. Cao and J. Gu, J. Mater. Chem. C, 2019, 7, 2725-2733.

57 H. N. Naziroglu and A. Sirit, Turk. J. Chem., 2012, 36, 659670.

58 T. Bui, S. Syed and C. F. Barbas III, J. Am. Chem. Soc., 2009, 131, 8758-8759.

59 B. T. Kumpuga and S. Itsuno, J. Catal., 2018, 361, 398-406. 60 G. Gao, X.-F. Bai, H.-M. Yang, J.-X. Jiang, G.-Q. Lai and L.-W. Xu, Eur. J. Org. Chem., 2011, 5039-5046. 